\title{
Estrutura e grupos ecológicos de um remanescente florestal urbano com histórico de perturbação recente em Uberlândia, MG
}

\author{
Sérgio de Faria Lopes ${ }^{{ }^{*}}$ \\ Vagner Santiago do Vale ${ }^{2}$ \\ Jamir Afonso do Prado Júnior ${ }^{2}$ \\ Ana Paula de Oliveira ${ }^{2}$ \\ Ivan Schiavini ${ }^{2}$ \\ ${ }^{1}$ Departamento de Biologia Geral, Instituto de Ciências Biológicas, \\ Universidade Estadual da Paraíba Rua Baraúnas, 352, CEP 584215-00, Campina Grande - PB, Brasil \\ ${ }^{2}$ PPG em Ecologia e Conservação de Recursos Naturais \\ Universidade Federal de Uberlândia, Uberlândia - MG, Brasil \\ *Autor para correspondência \\ defarialopes@gmail.com
}

Submetido em 20/04/2012

Aceito para publicação em 03/09/2012

\section{Resumo}

Avaliar a diversidade biológica contida nos atuais remanescentes urbanos e compreender a estrutura da comunidade e os seus processos ecológicos são importantes instrumentos para a conservação desses frágeis ecossistemas. Este estudo foi realizado em um fragmento de floresta estacional semidecidual, sujeito a longo histórico de perturbação antrópica. Foram amostrados todos os indivíduos arbóreos com circunferência à altura do peito $(\mathrm{CAP}) \geq 15 \mathrm{~cm}$, presentes em 25 parcelas $(20 \times 20 \mathrm{~m})$. As espécies foram classificadas em grupos sucessionais e quanto à síndrome de dispersão. Foram registrados 958 indivíduos, distribuídos em 69 espécies, pertencentes a 35 famílias. O grupo de espécies secundárias iniciais se destacou em todos os parâmetros fitossociológicos, seguido pelo das secundárias tardias. Tal resultado sugere que a floresta estudada se encontra num estádio intermediário de desenvolvimento sucessional. As frequentes e intensas perturbações antrópicas causadas pela retirada periódica da serapilheira e de plântulas dos compartimentos arbóreo, herbáceo e arbustivo, com a finalidade de permitir o uso da área para lazer e recreação, está comprometendo a regeneração das espécies e, assim, dificultando o lento processo de re-estruturação do remanescente em direção a fases mais maduras do processo de sucessão.

Palavras-chave: Diversidade florística; Fitossociologia; Floresta Estacional Semidecidual; Fragmentação florestal; Grupos sucessionais

\section{Abstract}

Structure and ecological groups of an urban, recently disturbed, forest remnant in Uberlândia, MG. The goal of this work was to evaluate the biological diversity in an urban forest remnant to better understand the structure of the community and ecological processes that are important for the conservation of these fragile ecosystems. The study was carried out in a fragment of seasonal semideciduous forest, with a long history of 
human disturbance, which is located in an urban area of Uberlândia, Minas Gerais. Samples from 25 (20x20m) plots were assessed, which included all individuals with a circumference at breast height $(\mathrm{CBH}) \geq 15 \mathrm{~cm}$. The species were classified based on successional group and dispersal syndrome. A total of 958 trees, from 69 species and 35 families, were recorded. The group of early secondary species presented higher values for all of the phytosociological parameters, followed by the late secondary species. The results suggest that the fragment is in an intermediate stage of successional development. Frequent and intense anthropogenic disturbance caused by the periodic removal of litter and tree, shrub and herb seedlings, to maintain an area for recreation, is compromising the regeneration of the species in this forest and thus hindering the slow process of regeneration of the remnant towards more mature stages of succession.

Key words: Floristic diversity; Forest fragmentation; Phytosociology; Seasonal Semideciduous Forest; Successional groups

\section{Introdução}

A fragmentação de habitats tem sido apontada como a mais grave ameaça à biodiversidade (COLLINGE, 1996). Consequentemente, a redução e modificação de habitats naturais tem norteado as investigações nas áreas da biologia da conservação e ecologia de paisagens (FISHER; LINDENMAYER, 2007). O crescimento urbano, um dos principais responsáveis pela fragmentação, implica cada vez mais em ações de planejamento adequadas na expansão dos grandes centros (DISLICH; PIVELLO, 2002).

Os estudos de áreas naturais afetadas pelo desenvolvimento das atividades humanas são importantes instrumentos para a manutenção desses frágeis ecossistemas (PEREIRA et al., 2001). A conservação destas áreas naturais, como bosques públicos, podem minimizar problemas em cidades caracterizadas pelo rápido e recente desenvolvimento, como poluição do ar e da água, impermeabilização do solo e elevação da temperatura (DISLICH; PIVELLO, 2002). Além disso, essas áreas naturais urbanas diminuem alguns fatores de perturbação, além de funcionarem como excelente local para o turismo ecológico, abrigo para a fauna e de manter o patrimônio fitogenético nativo (GUILHERME; NAKAJIMA, 2007). Por outro lado, estes remanescentes são comumente áreas pequenas e com acentuado isolamento, além de serem alvos de medidas de manejo inadequadas, como a introdução de espécies exóticas e nativas de outras regiões utilizadas normalmente para a arborização do entorno (SANTIN, 1999). Dessa forma, se fazem necessárias medidas adequadas de manejo para a conservação de seus atributos naturais.
Neste contexto, o histórico de perturbações tem muitas implicações na sucessão florestal, pois a intensidade das perturbações pode, dentre outros fatores, resultar em diferentes estruturas florestais secundárias (ALVES; METZGER, 2006). Estas florestas geralmente apresentam uma baixa densidade de árvores de maior porte e redução significativa na cobertura do dossel (TABARELLI; MANTOVANI, 1999; WILLIAMSLINERA, 2002). O aumento na descontinuidade do dossel e as variações das condições de luminosidade e estresse hídrico causados pela perturbação alteram o microclima da floresta (POORTER; BONGERS, 2006). Esta condição alterada afeta o estabelecimento e desenvolvimento das espécies secundárias e, assim, são esperadas diferenças não apenas quanto aos padrões florísticos destas florestas, mas também quanto aos processos relacionados às suas características ecológicas (ALVES; METZGER, 2006; POORTER; BONGERS, 2006).

Estudos que abordem a estrutura vertical, pouco investigados até o momento, devem ser tão importantes quanto aqueles relacionados à estrutura horizontal, considerando que a diferença na ocupação de nichos em estratos distintos é um dos fatores cruciais para a explicação da alta diversidade em florestas tropicais (TERBORGH, 1992). As relações entre os grupos de regeneração, de dispersão e de altura podem implicar em mudanças na composição das plantas ao longo do processo de sucessão florestal (TABARELLI; MANTOVANI, 1999).

O presente trabalho teve como objetivos: (a) apresentar as estruturas horizontal e vertical de um fragmento de Floresta Estacional Semidecidual, em 
ambiente urbano do município de Uberlândia, MG; (b) classificar as espécies em grupos ecológicos; (c) avaliar os efeitos de perturbações antrópicas, por meio desses grupos ecológicos, na estrutura do compartimento arbóreo e fornecer subsídios que visem contribuir para um plano de manejo adequado, que garanta a conservação desse relevante remanescente florestal.

\section{Material e Métodos}

\section{Área de estudo e histórico de perturbação}

O estudo foi conduzido no Parque Municipal do Sabiá, localizado na zona urbana, a $6 \mathrm{~km}$ do centro do município de Uberlândia, Minas Gerais. O Parque possui como coordenadas centrais $18^{\circ} 54^{\prime} 52^{\prime \prime} \mathrm{S}$ e $48^{\circ} 14^{\prime} 02^{\prime \prime} \mathrm{O}$, abrangendo uma área de 185 ha, numa altitude média de $890 \mathrm{~m}$. Dispõe em seu interior de um bosque com 35 ha de vegetação natural, apresentando diferentes formações naturais heterogêneas: floresta estacional semidecidual (objeto deste estudo), transição desta floresta com cerradão e mata de galeria (GUILHERME et al., 1998). A área do estudo em questão apresenta uma transição abrupta com a mata de galeria no seu limite inferior, e duas transições gradativas com manchas de cerradão, uma em cada limite da floresta (GUILHERME; NAKAJIMA, 2007).

A região onde está inserida o Parque apresenta clima Aw (KÖPPEN, 1948), caracterizada por apresentar invernos secos e verões chuvosos, com temperatura média anual de $22^{\circ} \mathrm{C}$ e índice pluviométrico de aproximadamente $1500 \mathrm{~mm} /$ ano (ROSA et al., 1991). O solo da região é classificado como Latossolo VermelhoAmarelo Álico (ROSA; SCHIAVINI, 2006).

O Parque do Sabiá foi criado em 1982 e, em 1997, foi reconhecido como uma Unidade de Conservação, enquadrada na categoria de Parque Municipal. Desde a criação do parque, as interferências feitas pelo poder público local foram basicamente voltadas para proporcionar melhores condições de lazer e recreação aos visitantes. O remanescente florestal foi manejado sem os cuidados necessários para que as áreas pudessem sofrer o mínimo de impactos possíveis, resultantes da intensa visitação pública. Até o ano de 1994, a administração do parque realizava a retirada da serapilheira e plântulas de espécies do estrato arbóreo e, também, do herbáceo e arbustivo, com a finalidade de garantir a segurança dos visitantes e permitir o uso da área para lazer e recreação.

Além disso, a existência de trilhas, passando pelo interior do remanescente, favoreceu a compactação do solo nas áreas mais frequentadas e o aumento da densidade populacional de uma espécie de bambu invasor (Olyra sp.) no estrato inferior. Estes eventos são impactos que podem estar comprometendo a estrutura e composição do estrato regenerativo e, assim, o estabelecimento dos indivíduos que irão compor a estrutura futura deste remanescente. Entretanto, desde 1994, outros impactos como limpeza periódica do sub-bosque foram suspensas e as interferências no remanescente têm sido menos impactantes. Também outras medidas foram adotadas no sentido de minimizar estes impactos, como a retirada de mesas e bancos da área e o isolamento do bosque por meio de cercas. Atualmente, a floresta do Parque do Sabiá apresenta sub-bosque com grande penetração de luz e com poucas lianas, epífitas e herbáceas.

\section{Levantamento dos dados}

Para o estudo fitossociológico em florestas tropicais, recomenda-se a utilização de área amostral de no mínimo 1 ha (FELFILI et al., 2005). Dessa forma, foram alocadas 25 parcelas contíguas e permanentes de $20 \times 20 \mathrm{~m}$. Em cada parcela, foram registrados e identificados todos os indivíduos arbóreos vivos e que apresentaram CAP (circunferência à altura do peito) $\geq 15 \mathrm{~cm}$. As medidas de altura foram tomadas com o auxílio do podão de coleta graduado de $14 \mathrm{~m}$ e por meio de estimativa visual comparativa para os indivíduos que ultrapassavam essa altura. Indivíduos que apresentavam troncos múltiplos tiveram as medidas de CAP tomadas individualmente. O material botânico coletado (amostras de folhas, flores e frutos) foi identificado por especialistas ou por comparação com coleções do herbário. O material testemunho para todas as espécies foi depositado no Herbarium Uberlandensis (HUFU) da Universidade Federal de Uberlândia, MG, durante o censo. As espécies foram classificadas em famílias, de acordo com as 
delimitações de APG III (2009) e os nomes das espécies foram conferidos com a base de dados disponíveis na página eletrônica do Missouri Botanical Garden (http:// www.mobot.org). O presente trabalho foi realizado durante o período de outubro a dezembro de 2006.

Os parâmetros fitossociológicos de densidade, dominância e frequência relativas e o de valor de importância (VI) foram estimados utilizando-se o programa FITOPAC 2.0 (SHEPHERD, 2009). Para a avaliação da diversidade alfa foi utilizado o índice de diversidade de Shannon (H') e equabilidade de Pielou (J').

\section{Estrutura vertical e grupos ecológicos}

A análise da estratificação da floresta foi realizada por espécie, para representar a real ocupação da mesma como função na comunidade (SMITH, 1973). Para isso, foram utilizadas apenas aquelas espécies que apresentaram no mínimo cinco indivíduos amostrados, eliminando da análise as espécies raras e aquelas de baixa densidade. A análise foi feita por estratos verticais, considerando-se três estratos arbóreos, delimitados segundo técnica não paramétrica de média e quartil utilizada por Vale et al. (2009).

As espécies consideradas na estrutura vertical foram classificadas em grupos sucessionais, cuja categorização foi feita tomando-se como base os trabalhos de Gandolfi et al. (1995), Tabarelli e Mantovani (1997), Pinard et al. (1999), Fonseca e Rodrigues (2000), Silva et al. (2003), Paula et al. (2004), Catharino et al. (2006), Oliveira Filho e Scolforo (2008), além de observações no campo, para auxiliar na classificação daquelas que, por ventura, não se enquadraram nos critérios propostos pelos autores consultados. Quando houve divergências da classificação entre os trabalhos seguiu-se a concordância da maioria destes e/ou observações de campo, com relação à ocorrência da espécie. As espécies foram distribuídas em três grupos, seguindo a classificação de Gandolfi et al. (1995), sendo eles: pioneira, secundária inicial e secundária tardia.

As espécies também foram classificadas quanto à síndrome de dispersão adotando os critérios morfológicos para diásporos definidos por Pijl (1982) e com auxílio da literatura (PINHEIRO; RIBEIRO, 2001). Dessa forma, os diásporos foram classificados em três grupos: anemocóricos (dispersão por vento), zoocóricos (dispersão por animais) e autocóricos (dispersão por gravidade e/ou explosiva).

\section{Resultados e Discussão}

No total foram amostradas 69 espécies, pertencentes a 31 famílias botânicas (Tabela 1). As famílias com maior riqueza foram Fabaceae (10 espécies), Rubiaceae e Vochysiaceae (cinco espécies cada). Quanto aos grupos sucessionais houve predominância de espécies secundárias iniciais em relação aos demais grupos (Tabela 2). Os maiores valores de densidade, frequência e VI pertenceram ao grupo das secundárias iniciais, que exerceram a maior influência na estrutura florestal. Budowski (1970) considera que o estádio sucessional de uma floresta é dado pelo grupo sucessional que apresentar maior proporção de indivíduos. Assim, os resultados sugerem que a floresta estudada se encontra em um estádio intermediário de desenvolvimento sucessional, podendo ser considerado como um remanescente de Floresta Estacional Semidecidual secundário quanto à sua conservação.

A utilização de parâmetros fitossociológicos é uma importante ferramenta para a caracterização do desenvolvimento florestal (FONSECA; RODRIGUES, 2000) e auxilia na classificação da floresta quanto ao seu estádio de desenvolvimento. Uma maior proporção de secundárias iniciais seguida de tardias sugere uma floresta sob pressão antrópica e em estádio intermediário (PAULA et al., 2004; PRADO JÚNIOR et al., 2010). Já quando ocorre uma maior proporção de pioneiras, pode-se inferir que é uma floresta com forte grau de perturbação (GUSSON et al., 2009), enquanto maiores proporções de tardias e baixa proporção de pioneiras demonstram uma área em avançado grau de maturidade e bom estádio de conservação (FONSECA; RODRIGUES, 2000; VALE et al., 2009). 
TABELA 1: Espécies arbóreas (CAP $\geq 15 \mathrm{~cm})$ amostradas na floresta Estacional Semidecidual do Parque do Sabiá, Uberlândia $(\mathrm{MG}) . \mathrm{NI}=$ número de indivíduos; $\mathrm{DR}=$ densidade relativa; DoR= dominância relativa; $\mathrm{FR}=$ frequência relativa; $\mathrm{VI}=$ valor de importância.

\begin{tabular}{|c|c|c|c|c|c|c|}
\hline Espécies & Famílias & NI & DR & DoR & FR & VI \\
\hline Tapirira obtusa (Benth.) J.D. Mitch. & Anacardiaceae & 80 & 8,35 & 8,8 & 3,68 & 20,83 \\
\hline Casearia grandiflora Cambess. & Salicaceae & 91 & 9,50 & 2,87 & 4,50 & 16,87 \\
\hline Callisthene major Mart. & Vochysiaceae & 36 & 3,76 & 9,5 & 2,66 & 15,91 \\
\hline Virola sebifera Aubl. & Myristicaceae & 67 & 6,99 & 4,73 & 3,89 & 15,61 \\
\hline Copaifera langsdorffii Desf. & Fabaceae & 14 & 1,46 & 10,11 & 2,45 & 14,03 \\
\hline Inga vera $\mathrm{DC}$. & Fabaceae & 60 & 6,26 & 2,61 & 3,89 & 12,76 \\
\hline Micropholis venulosa (Mart. \& Eichler) Pierre & Sapotaceae & 28 & 2,92 & 5,95 & 3,48 & 12,34 \\
\hline Cryptocarya aschersoniana $\mathrm{Mez}$ & Lauraceae & 27 & 2,82 & 5,77 & 3,27 & 11,86 \\
\hline Ocotea spixiana (Nees) Mez & Lauraceae & 16 & 1,67 & 6,12 & 3,07 & 10,86 \\
\hline Piptocarpha macropoda (DC.) Baker & Asteraceae & 41 & 4,28 & 2,62 & 3,27 & 10,17 \\
\hline Amaioua guianensis Aubl. & Rubiaceae & 49 & 5,11 & 1,85 & 3,07 & 10,04 \\
\hline Terminalia glabrescens (Cambess. ex A. St.-Hil.) Eichler & Combretaceae & 35 & 3,65 & 2,49 & 3,89 & 10,03 \\
\hline Astronium nelson-rosae Santin & Anacardiaceae & 28 & 2,92 & 3,15 & 3,48 & 9,55 \\
\hline Heisteria ovata Benth. & Olacaceae & 31 & 3,24 & 1,93 & 3,68 & 8,84 \\
\hline Lamanonia ternata Vell. & Cunoniaceae & 11 & 1,15 & 5,34 & 1,64 & 8,12 \\
\hline Aspidosperma discolor A. DC. & Apocynaceae & 17 & 1,77 & 3,92 & 2,04 & 7,74 \\
\hline Ixora brevifolia Benth. & Rubiaceae & 26 & 2,71 & 1,66 & 2,86 & 7,23 \\
\hline Miconia sellowiana Naudin & Melastomataceae & 22 & 2,30 & 1,72 & 2,86 & 6,88 \\
\hline Cheiloclinium cognatum (Miers) A.C. Sm. & Celastraceae & 23 & 2,40 & 0,47 & 3,07 & 5,93 \\
\hline Annona cacans Warm. & Annonaceae & 14 & 1,46 & 2,59 & 1,84 & 5,89 \\
\hline Duguetia lanceolata A. St.-Hil. & Annonaceae & 22 & 2,3 & 0,83 & 2,25 & 5,38 \\
\hline Qualea jundiahy Warm. & Vochysiaceae & 9 & 0,94 & 2,00 & 1,64 & 4,57 \\
\hline Apuleia leiocarpa (Vogel) J.F. Macbr. & Fabaceae & 8 & 0,84 & 1,91 & 1,64 & 4,38 \\
\hline Cupania vernalis Cambess. & Sapindaceae & 16 & 1,67 & 0,20 & 2,25 & 4,12 \\
\hline Schefflera morototoni (Aubl.) Maguire, Steyerm. \& Frodin & Araliaceae & 8 & 0,84 & 1,32 & 1,64 & 3,80 \\
\hline Xylopia aromatica (Lam.) Mart. & Annonaceae & 13 & 1,36 & 0,38 & 2,04 & 3,78 \\
\hline Maprounea guianensis Aubl. & Euphorbiaceae & 13 & 1,36 & 0,58 & 1,64 & 3,58 \\
\hline Cordia sellowiana Cham. & Boraginaceae & 11 & 1,15 & 0,62 & 1,64 & 3,41 \\
\hline Ocotea corymbosa (Meisn.) Mez & Lauraceae & 12 & 1,25 & 0,47 & 1,23 & 2,95 \\
\hline Hirtella glandulosa Spreng. & Chrysobalanaceae & 5 & 0,52 & 1,33 & 0,82 & 2,67 \\
\hline Ormosia arborea (Vell.) Harms & Fabaceae & 9 & 0,94 & 0,27 & 1,43 & 2,64 \\
\hline Cassia ferruginea Schrader ex DC. & Fabaceae & 4 & 0,42 & 0,67 & 0,82 & 1,91 \\
\hline Coussarea hydrangeifolia (Benth.) Müll. Arg. & Rubiaceae & 6 & 0,63 & 0,25 & 1,02 & 1,90 \\
\hline Myrcia splendens (Sw.) DC. & Myrtaceae & 6 & 0,63 & 0,13 & 1,02 & 1,77 \\
\hline Pseudolmedia laevigata Trécul & Moraceae & 6 & 0,63 & 0,1 & 1,02 & 1,75 \\
\hline
\end{tabular}




\begin{tabular}{|c|c|c|c|c|c|c|}
\hline Myrsine umbellata (Mart.) Mez & Primulaceae & 4 & 0,42 & 0,47 & 0,82 & 1,71 \\
\hline Vochysia tucanorum Mart. & Vochysiaceae & 4 & 0,42 & 0,67 & 0,61 & 1,70 \\
\hline Handroanthus impetiginosa (Mart. ex DC.) Standl. & Bignoniaceae & 4 & 0,42 & 0,28 & 0,82 & 1,52 \\
\hline Myrcia tomentosa (Aubl.) DC. & Myrtaceae & 5 & 0,52 & 0,1 & 0,82 & 1,44 \\
\hline Machaerium brasiliense Vogel & Fabaceae & 5 & 0,52 & 0,28 & 0,61 & 1,41 \\
\hline Senna macranthera (DC. ex Collad.) H.S. Irwin \& Barneby & Fabaceae & 5 & 0,52 & 0,06 & 0,82 & 1,40 \\
\hline Jacaranda macrantha Cham. & Bignoniaceae & 4 & 0,42 & 0,36 & 0,61 & 1,39 \\
\hline Qualea grandiflora Mart. & Vochysiaceae & 4 & 0,42 & 0,12 & 0,82 & 1,35 \\
\hline Pera glabrata (Schott) Poepp. ex Baill. & Euphorbiaceae & 4 & 0,42 & 0,08 & 0,82 & 1,31 \\
\hline Zanthoxylum rhoifolium Lam. & Rutaceae & 5 & 0,52 & 0,16 & 0,61 & 1,29 \\
\hline Margaritaria nobilis L. f. & Phyllanthaceae & 5 & 0,52 & 0,08 & 0,61 & 1,21 \\
\hline Dalbergia nigra (Vell.) Allemao ex Benth. & Fabaceae & 5 & 0,52 & 0,06 & 0,61 & 1,20 \\
\hline Styrax camporum Pohl & Styracaceae & 3 & 0,31 & 0,16 & 0,61 & 1,09 \\
\hline Xylopia sericea A. St.-Hil. & Annonaceae & 7 & 0,73 & 0,1 & 0,2 & 1,04 \\
\hline Vochysia magnifica Warm. & Vochysiaceae & 2 & 0,21 & 0,58 & 0,2 & 0,99 \\
\hline Siparuna guianensis Aubl. & Siparunaceae & 3 & 0,31 & 0,04 & 0,61 & 0,96 \\
\hline Bauhinia rufa (Bong.) Steud. & Fabaceae & 3 & 0,31 & 0,02 & 0,61 & 0,95 \\
\hline Diospyros hispida A. DC. & Ebenaceae & 2 & 0,21 & 0,26 & 0,41 & 0,88 \\
\hline Hirtella gracilipes (Hook. f.) Prance & Chrysobalanaceae & 2 & 0,21 & 0,19 & 0,41 & 0,80 \\
\hline Gomidesia lindeniana O. Berg & Myrtaceae & 2 & 0,21 & 0,05 & 0,41 & 0,67 \\
\hline Luehea grandiflora Mart. & Malvaceae & 2 & 0,21 & 0,04 & 0,41 & 0,66 \\
\hline Sweetia fruticosa Spreng. & Fabaceae & 2 & 0,21 & 0,03 & 0,41 & 0,65 \\
\hline Eugenia sp. & Myrtaceae & 1 & 0,10 & 0,16 & 0,20 & 0,46 \\
\hline Cedrela fissilis Vell. & Meliaceae & 1 & 0,10 & 0,15 & 0,20 & 0,46 \\
\hline Eriotheca pubescens (Mart. \& Zucc.) Schott. \& Endl. & Malvaceae & 1 & 0,10 & 0,06 & 0,20 & 0,37 \\
\hline Jacaranda cuspidifolia Mart. & Bignoniaceae & 1 & 0,10 & 0,05 & 0,20 & 0,36 \\
\hline Miconia affinis DC. & Melastomataceae & 1 & 0,10 & 0,03 & 0,20 & 0,34 \\
\hline Rudgea viburnoides (Cham.) Benth. & Rubiaceae & 1 & 0,10 & 0,03 & 0,20 & 0,34 \\
\hline Matayba guianensis Aubl. & Sapindaceae & 1 & 0,10 & 0,01 & 0,20 & 0,32 \\
\hline Protium heptaphyllum (Aubl.) Marchand & Burseraceae & 1 & 0,10 & 0,01 & 0,20 & 0,32 \\
\hline Faramea cyanea Müll. Arg. & Rubiaceae & 1 & 0,10 & 0,01 & 0,20 & 0,32 \\
\hline Coccoloba mollis Casar. & Polygonaceae & 1 & 0,10 & 0,01 & 0,20 & 0,32 \\
\hline Miconia cuspidata Mart. ex Naudin & Melastomataceae & 1 & 0,10 & 0,01 & 0,20 & 0,32 \\
\hline Roupala montana Aubl. & Proteaceae & 1 & 0,10 & 0,01 & 0,20 & 0,32 \\
\hline
\end{tabular}


O valor de importância acumulado para o grupo das pioneiras (Tabela 2) se deve a grande densidade e frequência relativas atingidas principalmente por Virola sebifera e Piptocarpha macropoda (Tabela 1). Esses dados demonstram o quão dispersas essas espécies estavam na área amostrada e podem ser explicadas pela presença de diversas pequenas clareiras, que favorece o estabelecimento das mesmas. Para as espécies secundárias tardias, as dominâncias relativas de Copaifera langsdorffi, Ocotea spixiana e Cryptocarya aschersoniana foram as responsáveis pelo elevado VI deste grupo (Tabela 1 e 2).

TABELA 2: Parâmetros fitossociológicos por grupo sucessional amostrado na Floresta Estacional Semidecidual do Parque do Sabiá, Uberlândia (MG), de espécies com número de indivíduos igual ou superior a cinco. $\mathrm{NI}=$ número de indivíduos; $\mathrm{DR}=$ densidade relativa; $\mathrm{FR}=$ frequência relativa; $\mathrm{DoR}=$ dominância relativa; VI \% = valor de importância relativa.

\begin{tabular}{lcccccc}
\hline \multicolumn{1}{c}{$\begin{array}{c}\text { Grupos } \\
\text { sucessionais }\end{array}$} & Espécies & NI & DR & FR & DoR & VI \% \\
\hline Pioneiras & 10 & 179 & 18,69 & 17,17 & 11,32 & 15,76 \\
Secundárias iniciais & 17 & 432 & 45,10 & 37,23 & 46,29 & 42,86 \\
Secundárias tardias & 14 & 286 & 29,84 & 33,75 & 37,82 & 33,81 \\
\hline
\end{tabular}

Em relação às síndromes de dispersão, pôde-se observar que a amostragem revelou uma composição de 41 espécies zoocóricas, 11 anemocóricas e duas autocóricas. Números similares foram encontrados para a abundância de indivíduos (Tabela 3 e 4), o que segue os padrões de florestas tropicais, onde mais de $50 \%$ das espécies produzem diásporos adaptados ao consumo de aves e mamíferos (HOWE; SMALLWOOD, 1982), assim como os padrões para florestas estacionais da região, em estado de conservação de médio a bom (DIAS NETO et al., 2009; VALE et al., 2009; PRADO JÚNIOR et al., 2010). Demonstra-se assim, o potencial das manchas de florestas estacionais presentes no Bioma Cerrado, em fornecer recursos para a manutenção da fauna o que aumenta a importância dessas florestas e os esforços destinados à conservação das mesmas, pois em florestas pouco preservadas há predomínio de grupos anemo-autocóricos (GUSSON et al., 2009).

TABELA 3: Espécies com cinco ou mais indivíduos amostrados nos três estratos da Floresta Estacional Semidecidual do Parque do Sabiá, Uberlândia (MG), com as respectivas características ecológicas. $\mathrm{NI}=$ número de indivíduos; $\mathrm{GS}=$ grupo sucessional; $\mathrm{P}=$ pioneira; $\mathrm{SI}=$ secundária inicial; $\mathrm{ST}$ = secundária tardia; $\mathrm{SB}=$ sub-bosque; $\mathrm{SD}=$ subdossel e $\mathrm{D}=$ dossel.

\begin{tabular}{lcccc}
\hline Espécie & NI & GS & Síndrome de dispersão & Estrato \\
\hline Amaioua guianensis & 49 & ST & Zoocórica & SB \\
Ixora brevifolia & 26 & ST & Zoocórica & SB \\
Cheiloclinium cognatum & 23 & ST & Zoocórica & SB \\
Cupania vernalis & 16 & SI & Zoocórica & SB \\
Ocotea corymbosa & 12 & ST & Zoocórica & SB \\
Coussarea hydrangeifolia & 6 & ST & Zoocórica & SB \\
Pseudolmedia laevigata & 6 & SI & Zoocórica & SB \\
Myrcia splendens & 6 & P & Zoocórica & SB \\
Dalbergia nigra & 5 & ST & Anemocórica & SB \\
Myrcia tomentosa & 5 & $\mathrm{P}$ & Zoocórica & SB \\
Casearia grandiflora & 91 & SI & Zoocórica & SD \\
Virola sebifera & 67 & P & Zoocórica & SD \\
Piptocarpha macropoda & 41 & $\mathrm{P}$ & Anemocórica & SD \\
\hline
\end{tabular}




\begin{tabular}{|c|c|c|c|c|}
\hline Terminalia glabrescens & 35 & $\mathrm{ST}$ & Anemocórica & SD \\
\hline Heisteria ovata & 31 & SI & Zoocórica & $\mathrm{SD}$ \\
\hline Duguetia lanceolata & 22 & ST & Zoocórica & SD \\
\hline Miconia sellowiana & 22 & $\mathrm{P}$ & Zoocórica & SD \\
\hline Maprounea guianensis & 13 & SI & Autocórica & SD \\
\hline Xylopia aromatica & 13 & $\mathrm{P}$ & Zoocórica & SD \\
\hline Cordia sellowiana & 11 & SI & Zoocórica & SD \\
\hline Ormosia arborea & 9 & SI & Zoocórica & SD \\
\hline Xylopia sericea & 7 & $\mathrm{P}$ & Zoocórica & SD \\
\hline Margaritaria nobilis & 5 & SI & Autocórica & SD \\
\hline Senna macranthera & 5 & $\mathrm{P}$ & Anemocórica & SD \\
\hline Zanthoxylum rhoifolium & 5 & $\mathrm{P}$ & Zoocórica & SD \\
\hline Tapirira obtusa & 80 & SI & Zoocórica & $\mathrm{D}$ \\
\hline Inga vera & 60 & SI & Zoocórica & $\mathrm{D}$ \\
\hline Callisthene major & 36 & SI & Anemocórica & $\mathrm{D}$ \\
\hline Astronium nelson-rosae & 28 & ST & Anemocórica & $\mathrm{D}$ \\
\hline Micropholis venulosa & 28 & SI & Zoocórica & $\mathrm{D}$ \\
\hline Cryptocarya aschersoniana & 27 & ST & Zoocórica & $\mathrm{D}$ \\
\hline Aspidosperma discolor & 17 & SI & Anemocórica & $\mathrm{D}$ \\
\hline Ocotea spixiana & 16 & $\mathrm{ST}$ & Zoocórica & $\mathrm{D}$ \\
\hline Annona cacans & 14 & ST & Zoocórica & $\mathrm{D}$ \\
\hline Copaifera langsdorffii & 14 & ST & Zoocórica & $\mathrm{D}$ \\
\hline Lamanonia ternata & 11 & SI & Anemocórica & $\mathrm{D}$ \\
\hline Qualea jundiahy & 9 & ST & Anemocórica & $\mathrm{D}$ \\
\hline Apuleia leiocarpa & 8 & SI & Anemocórica & $\mathrm{D}$ \\
\hline Schefflera morototoni & 8 & $\mathrm{P}$ & Zoocórica & $\mathrm{D}$ \\
\hline Hirtella glandulosa & 5 & SI & Zoocórica & $\mathrm{D}$ \\
\hline Machaerium brasiliense & 5 & SI & Anemocórica & $\mathrm{D}$ \\
\hline
\end{tabular}

TABELA 4: Síndromes de dispersão e grupos ecológicos na Floresta Estacional Semidecidual do Parque do Sabiá, Uberlândia (MG), de espécies com número de indivíduos igual ou superior a cinco. Os valores representam o número de espécies por grupo ecológico e, entre parênteses, o número de indivíduos correspondentes. Zoo $=$ zoocóricas, Anemo $=$ anemocóricas, Auto $=$ autocóricas, $\mathrm{ST}=$ secundárias tardias, $\mathrm{SI}=$ secundárias iniciais, $\mathrm{P}=$ pioneiras.

\begin{tabular}{lllllllc}
\hline \multicolumn{1}{c}{ Estrato } & \multicolumn{1}{c}{ Zoo } & Anemo & \multicolumn{1}{c}{ Auto } & \multicolumn{1}{c}{ ST } & \multicolumn{1}{c}{ SI } & \multicolumn{1}{c}{ P } & \multicolumn{1}{c}{ Total } \\
\hline Dossel & $9(252)$ & $7(114)$ & 0 & $6(108)$ & $9(250)$ & $1(8)$ & $16(366)$ \\
Subdossel & $10(278)$ & $3(81)$ & $2(18)$ & $2(57)$ & $6(160)$ & $7(160)$ & $15(377)$ \\
Sub-bosque & $9(149)$ & $1(5)$ & 0 & $6(121)$ & $2(22)$ & $2(11)$ & $10(154)$ \\
\hline Total & $28(679)$ & $11(200)$ & $2(18)$ & $14(286)$ & $17(432)$ & $10(179)$ & $41(897)$ \\
\hline
\end{tabular}


As espécies utilizadas na análise da estrutura vertical e síndrome de dispersão compõem $93 \%$ da densidade das árvores amostradas e a $92 \%$ do total do VI. Neste estudo, o dossel compreende espécies cujos indivíduos maduros atingem alturas superiores a $17 \mathrm{~m}$ e de sub-bosque abaixo de $12 \mathrm{~m}$. O estrato intermediário (subdossel) compreende espécies cujos maiores indivíduos se situam entre o dossel e sub-bosque, logo entre 12 e $17 \mathrm{~m}$. A maioria dos indivíduos amostrados (54\%) possui até $12 \mathrm{~m}$ de altura, que coincide com o sub-bosque da comunidade. Como esta metodologia de estratificação é baseada na classificação das espécies quanto à ocupação na estrutura vertical da floresta, muitos indivíduos pertencentes às espécies dos estratos superiores, apesar de possuírem altura inferior à mediana da comunidade, não foram considerados como pertencentes ao sub-bosque. De acordo com a metodologia, estes indivíduos representam os regenerantes destas espécies e, portanto, não representam a real ocupação da espécie na estratificação florestal (VALE et al., 2009). A partir desta altura, há um decréscimo progressivo em seu número à medida que as alturas aumentam até a máxima, que foi estimada em $35 \mathrm{~m}$. Habitualmente em florestas tropicais (pluviais e semidecíduas) são reconhecidos três estratos arbóreos (GUILHERME et al., 2004; VALE et al., 2009), mas a localização desses estratos e a sua estruturação variam nas diferentes áreas estudadas.

As espécies amostradas no dossel apresentam indivíduos em todos os estratos. Neste estrato, oito espécies são secundárias iniciais, seis secundárias tardias e uma pioneira (Schefflera morototoni - Tabela 3). O maior número de espécies anemocóricas (64\%) comprova a importância desta característica para ambientes sob condições de forte estacionalidade e sua predominância no dossel confirma a necessidade destas espécies em atingir elevadas alturas, para aumentarem a eficiência da dispersão de propágulos pelo vento. A ausência de espécies pioneiras (há apenas uma neste estrato) não é surpreendente. Apesar de existirem espécies pioneiras de vida longa (GOURLET-FLEURY et al., 2005), sua ocupação no dossel é dificultada pela competição com espécies tardias de vida longa, e melhores competidoras.

O maior número de espécies (15 espécies) foi amostrado no segundo estrato da floresta (subdossel).
Destas, seis são classificadas como secundárias iniciais, sete pioneiras e duas secundárias tardias (Tabela 4). As condições intermediárias de luz devem favorecer diferentes grupos de espécies (VALE et al., 2009), e a presença de muitas pioneiras e secundárias iniciais demonstram que o dossel da comunidade apresenta muitos espaços descontínuos que favorecem espécies demandantes de luz. Tal processo provavelmente seja reflexo do manejo pouco ecológico adotado na área antes de 1994, cujo desbaste de indivíduos deve ter favorecido o estabelecimento de espécies como Piptocarpha macropoda, Senna macranthera e Virola sebifera. O estudo realizado por Salles e Schiavini (2007), no mesmo remanescente, demonstrou que Piptocarpha macropoda e Xylopia aromatica apresentaram grande quantidades de indivíduos regenerantes, 187 e 65, respectivamente. Contudo, em um estudo de dinâmica da comunidade arbórea, realizado por Rosa (dados não publicados), nesta mesma área de estudo, indica que Piptocarpha macropoda apresentou alta taxa de mortalidade na comunidade, sugerindo seu caráter pioneiro. A mortalidade de indivíduos de grande porte de espécies pioneiras, bem como, alto valor no recrutamento, sugere a automanutenção da comunidade florestal, segundo o processo de sucessão (WHITMORE, 1990). O subdossel é predominantemente zoocórico (Tabela 4), fato importante, pois dispersores podem ocupar diferentes camadas verticais da floresta (PEARSON, 1971; SMITH, 1973; CLARK; POULSEN, 2001).

No sub-bosque, foram amostradas 10 espécies $(24,4 \%)$ (Tabela 4). Destas, seis são secundárias tardias, duas pioneiras e duas espécies secundárias iniciais (Tabela 4). Estes dados diferenciam dos encontrados por Paula et al. (2004) em um fragmento de floresta semidecidual em Viçosa, onde o sub-bosque, grupo com maior número de espécies, foi o das secundárias iniciais. Entretanto, corrobora os dados de um estudo de Vale et al. (2009), em Araguari (MG), cujo sub-bosque foi predominantemente composto por secundárias tardias. A presença de poucas espécies pioneiras neste estrato indica que o estádio de sucessão do remanescente não pode ser considerado inicial, uma vez que a baixa riqueza e densidade desse grupo sugere baixa regeneração (PAULA et al., 2004). Além disso, a presença e grande número de espécies secundárias tardias (Tabela 3 ) 
demonstram que a proteção na área, realizada a partir de 1994, está influenciando positivamente na regeneração da floresta e, medidas como cercamento e não uso direto por humanos é relevante para que a comunidade avance na série sucessional.

Neste estrato, merece destaque Dalbergia nigra, declarada como oficialmente ameaçada de extinção pela Portaria $\mathrm{n}^{\mathrm{o}}-37-\mathrm{N}$, de 03/04/92 do IBAMA, a qual apresenta cinco indivíduos arbóreos amostrados. Esta espécie, típica de dossel em Mata Atlântica (SILVA et al., 2003), foi introduzida no Parque do Sabiá para promover a arborização das áreas gramadas. Entretanto, suas sementes estão sendo dispersas até a floresta nativa, onde encontraram ambiente favorável para seu estabelecimento (SALLES; SCHIAVINI, 2007). Esta espécie pode ser considerada invasora local e deve ser manejada, para não prejudicar a flora nativa.

Em relação às síndromes de dispersão, todas as espécies neste estrato, com exceção de Dalbergia nigra, foram classificadas como zoocóricas (Tabela 3 e 4). Dalbergia nigra, espécie anemocórica, foi incluída neste estrato devido à altura de todos seus indivíduos (menor que $10 \mathrm{~m}$ ), provavelmente estes indivíduos possam ser considerados jovens, mas sabe-se que esta espécie atinge os estratos mais altos da floresta (SILVA et al., 2003). A presença de indivíduos anemocóricos nos estratos intermediários e inferiores indica que tais indivíduos ainda não alcançaram sua condição máxima de ocupação vertical na comunidade (CAIN et al., 1956), já que a dispersão pelo vento aumenta com a altura da árvore (PIRES-O'BRIEN; O'BRIEN, 1995) e por isso, espécies anemocóricas são comumente encontradas no dossel (NUNES et al., 2003).

Cada estrato da floresta possui características e composição florística distintas, pois as diversas espécies contêm indivíduos que estão passando por diferentes fases de desenvolvimento, isto é, morrendo, crescendo ou regenerando-se e, assim, uma parte das árvores dos estratos inferiores pertence a espécies cujos adultos potencialmente alcançam os estratos superiores (CAIN et al., 1956). As principais espécies do dossel estão bem representadas nos estratos inferiores. Entretanto, essas espécies apresentam a maior parte de seus indivíduos com alturas maiores ou iguais a $17 \mathrm{~m}$.
Em um estudo realizado na mesma área com o estrato regenerativo, as espécies secundárias tardias mais importantes (Tapirira obtusa, Cryptocarya aschersoniana e Copaifera langsdorffii) não tem tido sucesso regenerativo dentro da comunidade (SALLES; SCHIAVINI, 2007), o que poderá significar a extinção local destas espécies, devido à ausência de recrutamento, e resultar na mudança florística da formação (NASCIMENTO et al., 1999). Entretanto, Ocotea corymbosa, classificada como secundária tardia, foi a espécie com maior número de indivíduos no estrato regenerativo (189 indivíduos), ou seja, sua presença em $83 \%$ das parcelas no estrato regenerativo permite considerar que essa espécie tem encontrado um ambiente favorável para o seu estabelecimento.

A análise dos resultados para os grupos ecológicos nos estratos demonstra que o remanescente estudado se encontra em estádio secundário de sucessão, devido aos altos valores fitossociológicos encontrados para as espécies secundárias iniciais, em todos os estratos, e à baixa representatividade de espécies pioneiras e secundárias tardias (Tabela 4), o que está diretamente relacionado ao histórico de perturbações (GANDOLFI et al., 1995). As perturbações provocadas no remanescente apenas afetaram diretamente o processo de regeneração natural (SALLES; SCHIAVINI, 2007), principalmente pela formação de clareiras naturais ou antrópicas, que tem proporcionado o estabelecimento natural de espécies pioneiras e o crescimento de grupos regenerantes.

A análise da estrutura vertical realizada qualitativamente (por meio da identificação de quais espécies pertencem a cada um dos estratos verticais do remanescente) permitiu detectar mais claramente não apenas os padrões florísticos de cada estrato vertical, mas também compreender os processos ecológicos destes estratos, relacionados às suas características funcionais das espécies (Tabela 4). Ao que tudo indica, os estratos identificados estão em acordo com o esperado na estrutura de uma floresta semidecídua. O sub-bosque do remanescente é formado em sua maioria por espécies secundárias tardias e zoocóricas, enquanto o dossel é constituído principalmente por espécies secundárias tardias de grande crescimento, como Apuleia leiocarpa e Copaifera langsdorffii, e espécies 
anemocóricas (Astronium nelson-rosae e Callisthene major). Segundo Roth (1987), espécies com síndromes de dispersão zoocóricas, com frutos e sementes pesadas, predominariam nos estratos mais baixos da floresta, nos quais a vida animal é mais intensa, e espécies com síndromes autocóricas e anemocóricas predominariam nos estratos superiores de florestas tropicais. Dessa forma, o tipo de dispersão de diásporos é diferenciada entre os estratos em florestas tropicais (KILLEN et al., 1998), propiciando benefícios e disponibilidade de recursos distintos entre os estratos na comunidade.

Os resultados encontrados na floresta estacional semidecidual do Parque do Sabiá reforçam a relevância de estudos realizados em remanescentes urbanos, tendo permitido demonstrar que níveis de perturbação antrópicas resultantes de medidas de manejo inadequadas afetam diretamente o processo de regeneração natural, o que poderá mudar a composição e estrutura horizontal futura, sem, contudo, afetar diretamente a estrutura vertical atual.

\section{Referências}

ALVES, L. F.; METZGER, J. P. A regeneração florestal em áreas de floresta secundária na Reserva Florestal do Morro Grande, Cotia, SP. Biota Neotropica, Campinas, v. 6, p. 1-26, 2006.

APG III - ANGIOSPERM PHYLOGENY GROUP. An update of the Angiosperm Phylogeny Group classification for the orders and families of flowering plants: APG III. The Linnean Society of London. Botanical Journal of the Linnean Society, London, v. 161, p. 105-121, 2009.

BUDOWSKI, G. The distinction between old secondary and climax species in tropical central american lowland rainforest. Tropical Ecology, Varanasi, v. 11, p.44-48, 1970.

CAIN, S. A.; CASTRO, G. M. O.; PIRES, J. M.; SILVA, N. T. Application of some phytosociological techniques to Brazilian rain forest. American Journal of Botany, St. Louis, v. 43, p. 911-941, 1956.

CATHARINO, E. L. M.; BERNACCI, L. C.; FRANCO, G. A. D. C.; DURIGAN, G.; METZGER, J. P. Aspectos da composição e diversidade do componente arbóreo das florestas da Reserva Florestal do Morro Grande, Cotia, SP. Biota Neotropica, Campinas, v. 6, p. 1-24, 2006.

CLARK, C. J.; POULSEN, J. R. The role of arboreal seed dispersal groups on the seed rain of a lowland tropical forest. Biotropica, Washington, v. 33, p. 606-620, 2001.

COLLINGE, S. K. Ecological consequences of habitat fragmentation: implications for landscape architecture and planning. Landscape and Urban Planning, San Diego, v. 36, p. 59-77, 1996.
DIAS NETO, O. C.; SCHIAVINI, I.; LOPES, S. F.; VALE, V. S.; GUSSON, A. E.; OLIVEIRA, A. P. Estrutura fitossociológica e grupos ecológicos em fragmento de floresta estacional semidecidual, Uberaba, Minas Gerais, Brasil. Rodriguésia, Rio de Janeiro, v. 60, p. 1087-1100, 2009.

DISLICH, R.; PIVELLO, V. R. Tree structure and species composition changes in an urban tropical forest fragment (São Paulo, Brazil) during a five-years interval. Boletim Botânica da Universidade de São Paulo, São Paulo, v. 20, p. 1-11, 2002.

FELFILI, J. M.; CARVALHO, F. A.; HAIDAR, R. F. Manual para o monitoramento de parcelas permanentes nos biomas Cerrado e Pantanal. Brasília: Departamento de Engenharia Florestal. Universidade de Brasília. 2005. 51 p.

FISCHER, J.; LINDENMAYER, D.B. Landscape modification and habitat fragmentation: a synthesis. Global Ecology and Biogeography, Ottawa, v.16, p. 265-280, 2007.

FONSECA, R. C. B.; RODRIGUES. R. R. Análise estrutural e aspectos do mosaico sucessional de uma floresta semidecídua em Botucatu, SP. Sc. Florestalis, Piracicaba, v. 57, p. 27-43, 2000.

GANDOLFI, S.; LEITÃO-FILHO, H. F.; BEZERRA, C. L. F. Levantamento florístico e caráter sucessional das espécies arbustivo-arbóreas de uma floresta mesófila semidecídua no município de Guarulhos, SP. Revista Brasileira de Biologia, São Paulo, v. 55, p. 753-767, 1995.

GOURLET-FLEURY, S.; CORNU, G.; JÉSEL, S.; DESSARD, H.; JOURGET, J.; BLANC, L.; PICARD, N. Using models to predict recovery and assess tree species vulnerability in logged tropical forests: A case study from French Guiana. Forest Ecology and Management, Amsterdam, v. 209, p. 69-85, 2005

GUARIGUATA, M. R.; OSTERTAG, R. Neotropical secondary forest succession: changes in structural and functional characteristics. Forest Ecology and Management, Amsterdam, v. 148, p. 185-206, 2001.

GUIlHerme, F. A. G.; MOREllato, L. P. C.; ASSIS, M. A. Horizontal and vertical tree community structure in a section of lowland Atlantic Rain Forest in the Intervales State Park, southeastern Brazil. Revista Brasileira de Botânica, São Paulo, v. 27, n. 4, p. 725-737, 2004.

GUILHERME, F. A. G.; NAKAJIMA, J. N. Estrutura da vegetação arbórea de um remanescente ecotonal urbano floresta-savana no Parque do Sabiá, em Uberlândia, MG. Revista Árvore, Viçosa, v. 31, p. 329-338, 2007.

GUILHERME, F. A. G.; NAKAJIMA, J. N.; LIMA, C. A. P.; VANINI, A. Fitofisionomias e flora lenhosa nativa do Parque do Sabiá, Uberlândia, MG. Daphne, Belo Horizonte, v. 8, p. 17-30, 1998.

GUSSON, A. E.; LOPES, S. F.; DIAS NETO, O. C.; VALE, V. S.; OLIVEIRA, A. P.; SCHIAVINI, I. Características químicas do solo e estrutura de um fragmento de floresta estacional semidecidual em Ipiaçu, Minas Gerais, Brasil. Rodriguésia, Rio de Janeiro, v. 60, p. 403-414, 2009.

HOWE, H. F.; SMALLWOOD, J. Ecology of seed dispersal. Annual Review of Ecology and Systematics, Palo Alto, v. 13, p. 201-228, 1982.

KILLEEN, T.; JARDIM, A.; MANAMI, F.; SARAVIA, P.; ROJAS, N. Diversity, composition, and structure of a tropical deciduous 
forest in the Chiquitania region of Santa Cruz, Bolivia. Journal of Tropical Ecology, Winchelsea, v. 14, p. 803-827, 1998.

KÖPPEN, W. Climatologia: con un estudio de los climas de la tierra. Trad. P.R. Hendrichs Pérez. México: Fondo de Cultura Economica, 1948. 479 p.

NASCIMENTO, H. E. M.; DIAS, A. S.; TANABEZ, A. A. J.; VIANA, V. M. Estrutura e dinâmica de populações arbóreas de um fragmento de Floresta Estacional Semidecidual na região de Piracicaba, São Paulo. Revista Brasileira de Botânica, São Paulo, v. 59, p. 329-342, 1999.

NUNES, Y. R.; MENDONÇA, A. V. R. BOTEZELLI, L.; MACHADO, E. L. M.; OLIVEIRA-FILHO, A. T. Variações da fisionomia, diversidade e composição de guildas da comunidade arbórea em um fragmento de floresta semidecidual em lavras, MG. Acta Botanica Brasilica, Feira de Santana, v. 17, p. 213- 229, 2003.

OLIVEIRA-FILHO, A. T.; SCOLFORO, J. R. S. (Org.). Inventário florestal de Minas Gerais: espécies arbóreas da flora nativa. Lavras: Editora UFLA, 2008. 620 p.

PAULA, A.; SILVA, A. F.; MARCO-JÚNIOR, P.; SANTOS, F. A. M.; SOUZA; A. L. Sucessão ecológica da vegetação arbórea em uma floresta estacional semidecidual, Viçosa, MG, Brasil. Acta Botanica Brasilica, Feira de Santana, v. 18, p. 407-423, 2004.

PEARSON, D. L. Vertical stratification of birds in a tropical dry forest. The Condor, Albuquerque, v. 73, p. 46-55, 1971.

PEREIRA, I. M.; ANDRADE, L. A.; COSTA, J. R. M.; DIAS; J. M. Regeneração natural em um remanescente de Caatinga sob diferentes níveis de perturbação, no Agreste Paraibano. Acta Botanica Brasilica, Feira de Santana, v. 15, p. 413-426, 2001.

PINARD, M. A.; PUTZ, F. E.; RUMIZ, D.; GUZMÁN, R.; JARDIM, A. Ecological characterization of tree species for guiding forest management decisions in seasonally dry forests in Lomerío, Bolivia. Forest Ecology and Management, Amsterdam, v. 113, p. 201-213, 1999.

PIRES-O'BRIEN, M. J.; O'BRIEN, C. M. Ecologia e modelamento de florestas tropicais. Belém: Faculdade de Ciências Agrárias do Pará. 1995. 400 p.

PINHEIRO, F.; RIBEIRO, J. F. Síndromes de dispersão em matas de galeria do Distrito Federal. In: RIBEIRO, J. F.; FONSECA, C. E . L.; SOUSA-SILVA, J. C. (Ed.). Cerrado: caracterização e recuperação de matas de galeria. Planaltina: Embrapa Cerrados; Brasília: Embrapa Informação Tecnológica, 2001, p. 315-328.

PIJL, V. D. L. Principles of dispersal in higher plants. 3 ed. New York: Springer Verlag, 1982. 402 p.

POORTER, L.; BONGERS, F. Leaf traits are good predictors of plant performance across 53 rain forest species. Ecology, New York, v. 87, p. 1733-1743, 2006.

PRADO JÚNIOR, J. A.; VALE, V. S.; OLIVEIRA, A. P.; GUSSON, A. E.; DIAS NETO, O. C.; LOPES, S. F.; SCHIAVINI, I. Estrutura da comunidade arbórea em um fragmento de floresta estacional semidecidual localizada na reserva legal da Fazenda Irara, Uberlândia, MG. Bioscience Journal, Uberlândia, v. 26, p. 638-647, 2010.
ROSA, A. G.; SCHIAVINI, I. Estrutura da comunidade arbórea em um remanescente florestal urbano (Parque do Sabiá, Uberlândia, MG). Bioscience Journal, Uberlândia, v. 22, p. 151-162, 2006.

ROSA, R.; LIMA, S. C.; ASSUNÇÃO, W. L. Abordagem preliminar das condições climáticas de Uberlândia (MG). Sociedade e Natureza, Uberlândia, v. 3, p. 91-108, 1991.

SALLES, J. C.; SCHIAVINI, I. Estrutura e composição do estrato de regeneração em um fragmento florestal urbano: implicações para a dinâmica e a conservação da comunidade arbórea. Acta Botanica Brasilica, Feira de Santana, v. 21, p. 223-233, 2007.

SANTIN, D. A. A vegetação remanescente do município de Campinas (SP): mapeamento, caracterização fisionômica e florística, visando a conservação. 1999. 467 f. Tese (Doutorado em Ciências Biológicas) - Universidade Estadual de Campinas, Campinas. 1999.

SHEPHERD, G. J. Fitopac 2.01 - Manual do usuário. Campinas: UNICAMP, 2009. Versão eletrônica.

SILVA, A. F.; OLIVEIRA, R. V.; SANTOS, N. R. L.; PAULA, A. Composição florística e grupos ecológicos das espécies de um trecho de floresta semidecídua submontana da Fazenda São Geraldo, Viçosa-MG. Revista Árvore, Viçosa, v. 27, p. 311-319, 2003.

SMITH, A. P. Stratification of temperature and tropical forests. The American Naturalist, Chicago, v. 107, p. 671-683, 1973.

TABARELLI, M.; MANTOVANI. W. Colonização de clareiras naturais na floresta atlântica no sudeste do Brasil. Revista Brasileira de Botânica, São Paulo, v. 20, p. 57-66, 1997.

TABARELLI, M.; MANTOVANI. W. A regeneração de uma floresta tropical montana após corte e queima (São Paulo-Brasil). Revista Brasileira de Botânica, São Paulo, v. 22, p. 217-223, 1999.

TERBORGH, J. Diversity and the tropical rain forest. New York: Scientific American Library, 1992. 242 p.

VALE, V. S.; SCHIAVINI, I.; LOPES, S. F.; DIAS NETO, O. C.; OLIVEIRA, A. P.; GUSSON, A. E. Composição florística e estrutura do componente arbóreo em um remanescente primário de floresta estacional semidecidual em Araguari, Minas Gerais, Brasil. Hoehnea, São Paulo, v. 36, p. 417-429, 2009.

WHITMORE, T. C. An introdution to the tropical rain forests. Clarendon Press, Oxford. 1990. 198 p.

WILLIAMS-LINERA, G. Tree species richness complementarity, disturbance and fragmentation in a Mexican tropical montane cloud forest. Biodiversity and Conservation, New York, v. 11, p. 1825$1843,2002$. 